\title{
Resolved double-lined spectroscopic binaries: A neglected source of hypothesis-free parallaxes and stellar masses
}

\author{
D. Pourbaix ${ }^{\star} \star \star \star, 1,2$ \\ 1 Institut d'Astronomie et d'Astrophysique, Université Libre de Bruxelles, CP. 226, Boulevard du Triomphe, B-1050 Bruxelles, \\ Belgium \\ 2 Royal Observatory of Belgium, Avenue Circulaire 3, B-1180 Bruxelles, Belgium
}

Received September 21, 1999; accepted May 9, 2000

\begin{abstract}
Double-lined spectroscopic binaries, once visually resolved (VB-SB2), provide hypothesis-free orbital parallaxes and masses of both components. Unlike eclipsing-spectroscopic binaries for which many accurate masses are already known (Andersen 1991; Andersen 1997), the number of VB-SB2 remains rather small. This paper presents 40 such systems for which published visual observations and radial velocities allow a simultaneous adjustment of both data sets. The precision of the individual masses as well as the evolution of that precision with respect to the published precision is investigated.
\end{abstract}

Key words: methods: data analysis — stars: binaries: spectroscopic, visual; distances; fundamental parameters

\section{Introduction}

In his review on stellar masses, Popper (1980) quoted only five resolved spectroscopic binaries (and three additional for which the individual masses could become available in a close future). Thanks to speckle observation programmes (e.g., McAlister 1997), many spectroscopic binaries have been resolved for twenty years. Furthermore, new interferometers (NPOI (Armstrong et al. 1998), TPI (Colavita et al. 1999)) have already shown their capabilities by providing the visual orbit of short period binaries (Malbet et al. 1998; Koresko et al. 1998; Boden et al. 1999).

When a spectroscopic binary is visually resolved, the usual approach for deriving the parallax and masses consists in combining the independently obtained visual and

\footnotetext{
Send offprint requests to: D. Pourbaix,

e-mail: pourbaix@astro.ulb.ac.be

* ESA-PRODEX contract P171912 \& 13318/98/NL/VJ.

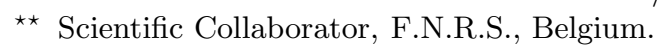

spectroscopic orbits. Such an approach completely disregards the potential correlation between some orbital parameters (Pourbaix \& Eichhorn 1999). In order to obtain uncertainties for the masses and parallax fully consistent with the precision of both types of observations, it is worth fitting the two sets simultaneously (Pourbaix 1998).

In Sect. 2, we list 40 binaries whose relative positions and radial velocities of both components have been published. When available, we also give the photometric and spectroscopic characteristics of the components. The evolution of the precision of the masses with respect to the previously published values thanks to the simultaneous fit is investigated in Sect. 3.

\section{Resolved spectroscopic binaries}

Table 1 lists the stars identified as VB-SB2. Columns $m_{v 1}$ and $m_{v 2}$ contain the apparent visual magnitudes of both components. When the second column is blank, it is likely that the first one actually represents the overall visual magnitude of the binary. Columns $S p_{1}$ and $S p_{2}$ give the spectral types of both components. When only one spectrum is given, it is usually the overall system spectrum.

The column "Type" identifies how the spectra were actually observed and processed. SB2 designates systems whose spectra of both components are clearly differentiable and the radial velocities can be measured without any supplementary assumption. LWSB (Line-Width Spectroscopic Binary; Duquennoy \& Mayor (1991)) are systems whose spectra are never individually separated. In order to disentangle them and obtain the two radial velocities, some extra assumptions (e.g., line profiles) were necessary. The orbital parallax of LWSB and, therefore, the derived masses can be very sensitive to the Gaussians used to disentangle the spectra (e.g. HIP 75312). The column "Ref" gives the references of the magnitudes and spectral types as well as all observations used for the adjustments. 
Table 1. List of resolved double-lined spectroscopic binaries

\begin{tabular}{|c|c|c|c|c|c|c|c|c|}
\hline HIP & Name & HD & $\begin{array}{c}m_{v 1} \\
(\mathrm{mag})\end{array}$ & $S p_{1}$ & $\begin{array}{c}m_{v 2} \\
(\mathrm{mag})\end{array}$ & $S p_{2}$ & Type & Ref \\
\hline 677 & $\alpha$ And & 358 & 2.22 & B8IV & 4.21 & & SB2 & $1,30,32,33$ \\
\hline 2941 & ADS 520 & 3443 & 5.57 & G8V & & G8V & LWSB & $2,26,31,34$ \\
\hline 4463 & $\eta$ And & 5516 & 4.42 & G8III-IV & & & SB2 & 3,35 \\
\hline 7580 & Kui 7 & 10009 & 6.54 & F5V & 7.77 & & SB2 & 4,36 \\
\hline 8903 & $\beta$ Ari & 11636 & 2.64 & $\mathrm{~A} 5 \mathrm{~V}$ & & & SB2 & $5,64,65,67$ \\
\hline 10064 & $\beta$ Tri & 13161 & 3.0 & A5III & & & SB2 & $6,45,62,63$ \\
\hline 10644 & $\delta$ Tri & 13974 & 4.85 & G0V & & & LWSB & $1,45,27$ \\
\hline 10952 & $\Sigma 248$ & & 8.5 & $\mathrm{~K} 0 \mathrm{~V}$ & 10.5 & $\mathrm{~K} 0 \mathrm{~V}$ & SB2 & $7,8,34,61$ \\
\hline 12390 & $\epsilon$ Cet & 16620 & 4.84 & F5V & & $\mathrm{F} 6 \mathrm{~V}$ & LWSB & $1,27,37$ \\
\hline 12623 & 12 Per & 16739 & 4.91 & F9V & & & SB2 & $1,26,37,59,60$ \\
\hline 14328 & $\gamma$ Per & 18925 & 3.25 & G8III & 4.49 & $\mathrm{~A} 3 \mathrm{~V}$ & SB2 & 9,10 \\
\hline 20087 & $51 \mathrm{Tau}$ & 27176 & 5.64 & $\mathrm{~A} 8 \mathrm{~V}$ & & G0V & LWSB & 11,37 \\
\hline 20661 & Fin 342 & 27991 & 6.44 & F7V & & & SB2 & 12,37 \\
\hline 24608 & $\alpha$ Aur & 34029 & 0.08 & G8III & & G0III & SB2 & $13,14,57,58$ \\
\hline 28360 & $\beta$ Aur & 40183 & 1.96 & A2IV & & & SB2 & $1,45,56$ \\
\hline 38382 & 9 Pup & 64096 & 5.72 & $\mathrm{G} 2 \mathrm{~V}$ & 6.17 & & SB2 & $1,2,27,37$ \\
\hline 45170 & Fin $347 \mathrm{Aa}$ & 79096 & 6.49 & G8V & & G8V & SB2 & $15,37,49$ \\
\hline 46404 & HR 3750 & 81809 & 5.80 & $\mathrm{G} 2 \mathrm{~V}$ & 6.60 & G9V & LWSB & $2,27,34,37$ \\
\hline 57565 & 93 Leo & 102509 & 4.60 & G8III & & A7V & SB2 & 16,45 \\
\hline 65378 & $\zeta^{1} \mathrm{UMa}$ & 116656 & 2.27 & $\mathrm{~A} 1 \mathrm{VpSrSi}$ & & & SB2 & $1,54,55$ \\
\hline $71683 / 1$ & $\alpha$ Cen & $128620 / 1$ & -0.01 & $\mathrm{G} 2 \mathrm{~V}$ & 1.33 & $\mathrm{~K} 1 \mathrm{~V}$ & SB2 & 1 \\
\hline 73182 & Gl 570B & 131976 & 8.33 & $\mathrm{M} 1.5 \mathrm{~V}$ & 9.94 & M3V & LWSB & 17,43 \\
\hline 75312 & $\eta \mathrm{CrB} \mathrm{A}$ & $137107 / 8$ & 5.62 & $\mathrm{G} 2 \mathrm{~V}$ & 5.96 & $\mathrm{G} 2 \mathrm{~V}$ & LWSB & $2,26,34$ \\
\hline 85667 & $\Sigma 2173$ & 158614 & 6.02 & G9IV-V & 5.93 & G9IV-V & SB2 & $1,18,26,34,37$ \\
\hline 87895 & HR 6697 & 163840 & 6.39 & G0-2V & 9.19 & $\mathrm{~K} 2-5 \mathrm{~V}$ & SB2 & 19 \\
\hline 88601 & $70 \mathrm{Oph}$ & 165341 & 4.2 & $\mathrm{~K} 0 \mathrm{~V}$ & 6.0 & $\mathrm{~K} 5 \mathrm{~V}$ & SB2 & $20,34,51,52,53$ \\
\hline 89937 & $\chi$ Dra & 170153 & 3.57 & F7V & & & SB2 & $1,26,37,66$ \\
\hline 91636 & $\Sigma 2367$ & 172865 & 7.47 & G5IV & 7.97 & G5IV & SB2 & $21,22,34,50$ \\
\hline 95995 & Gl 762.1 & 184467 & 6.59 & $\mathrm{~K} 1 \mathrm{~V}$ & & & SB2 & $2,37,48,49$ \\
\hline 96683 & $\phi$ Cyg & 185734 & 5.31 & KOIII & 5.6 & KOIII & SB2 & $23,24,25,47$ \\
\hline 98416 & Gl 773.3 & 189340 & 5.88 & F8V & & & LWSB & $1,37,46$ \\
\hline 99376 & ADS 13461 & 191854 & 8.058 & G4V & 8.598 & G8V & LWSB & 26,34 \\
\hline 99473 & $\theta \mathrm{Aql}$ & 191692 & 3.23 & B9.5III & & & SB2 & $1,44,45$ \\
\hline 103655 & Gl 815AB & & 10.29 & $\mathrm{dM} 3 \mathrm{e}$ & 12.19 & & SB3 & $2,34,43$ \\
\hline 104858 & $\delta \mathrm{Equ}$ & 202275 & 5.25 & F8V & 5.25 & & SB2 & $2,27,37$ \\
\hline 104987 & $\alpha \mathrm{Equ}$ & $202447 / 8$ & 3.92 & G2III & & $\mathrm{A} 5 \mathrm{~V}$ & SB2 & $28,41,42$ \\
\hline 108917 & $\xi$ Cep A & 209790 & 4.29 & $\mathrm{~A} 3 \mathrm{~m}$ & & & SB2 & $1,37,40$ \\
\hline 111170 & Gl 862.1 & 213429 & 6.14 & F7V & & & LWSB & $1,37,39$ \\
\hline 111528 & ADS 16098 & 214222 & 8.4 & G0IV & & & SB2 & $4,34,37,49$ \\
\hline 114576 & ADS 16591 & 219018 & 8.4 & $\mathrm{G} 2 \mathrm{~V}$ & 8.6 & G4V & SB2 & $29,34,38$ \\
\hline
\end{tabular}

Ref. 1: Hoffleit \& Jaschek 1982; 2: Gliese 1969; 3: Hummel et al. 1993; 4: Tokovinin 1993; 5: Tomkin \& Tran 1987; 6: Cowley et al. 1969; 7: Carney et al. 1994; 8: Torres 1995; 9: Bahng 1958; 10: Griffin et al. 1994; 11: Torres et al. 1997a; 12: Torres et al. 1997b; 13: Rufener 1976; 14: Bagnuolo \& Sowell 1989; 15: Mason et al. 1996; 16: Batten et al. 1983; 17: Mariotti et al. 1990; 18: Batten et al. 1991; 19: McAlister et al. 1995; 20: Heintz 1988; 21: Christy \& Walker 1969; 22: Stephenson \& Sanwal 1969; 23: Fernie 1969; 24: Fekel 1992; 25: Armstrong et al. 1992; 26: Duquennoy et al. 1991; 27: Duquennoy \& Mayor 1988a; 28: Pike 1978; 29: Dommanget \& Nys 1982; 30: Pan et al. 1992; 31: Roman 1952; 32: Tomkin et al. 1995; 33: Aikman 1976; 34: Worley \& Douglass 1996; 35: Gordon 1946; 36: Hartkopf et al. 1996; 37: Hartkopf et al. 1997; 38: Duquennoy 1987; 39: Duquennoy et al. 1988; 40: Vickers \& Scarfe 1976; 41: Rosvick \& Scarfe 1991; 42: Armstrong et al. 1992; 43: Duquennoy \& Mayor 1988b; 44: Cesco \& Struve 1946; 45: Hummel et al. 1995; 46: Duquennoy \& Mayor 1991; 47: Rach \& Herbig 1961; 48: McClure 1983; 49: Mayor \& Udry (Priv. Comm.); 50: Batten et al. 1982; 51: Berman 1932; 52: Batten et al. 1984; 53: Batten \& Fletcher 1991; 54: Fehrenbach \& Prevot 1961; 55: Hummel et al. 1998; 56: Smith 1948; 57: Hummel et al. 1994; 58: Barlow et al. 1993; 59: Colacevich 1941; 60: Barlow et al. 1998; 61: Prieur et al. 2000; 62: Struve \& Pogo 1928; 63: Ebbighausen 1953; 64: Pan et al. 1990; 65: Gorza \& Heard 1971; 66: Tomkin et al. 1987; 67: Hilditch et al. 1988. 
A few systems are not analyzed even if they belong to the VB-SB2 category. The SB2 systems presented by Delfosse et al. (1999) are absent because neither the radial velocities nor the visual observations have been published yet. For the TPI systems (Malbet et al. 1998; Koresko et al. 1998; Boden et al. 1999), the nature of the visual observations is the reason why we disregarded them. Indeed, for the time being, our code cannot simultaneously fit radial velocities and optical interferometric raw data (i.e. visibilities). The reason for disregarding ADS 14396 is different. The way Griffin (1984) disentangled its spectra to obtain the individual radial velocities makes them very related to the visual orbit he used. We cannot therefore use them together with some visual observations and still claim we get bias-free results.

A few systems have already been described in details in previous papers: HIP 7580 (Pourbaix 1998), 14328 (Pourbaix 1999) and 71683/1 (Pourbaix et al. 1999). For all systems, we list the orbital parameters in Table 2 . A few of these binaries are described slightly further. (Dis)agreement is always stated on a $3 \sigma$ basis. If the two compared values are given with their own uncertainty, the agreement can be partial only whether the standard deviation of one result or the other is used. When the time interval is larger than the orbital period, the adopted periastron epoch is the one within that interval which maximizes the efficiency (Eichhorn 1989; Pourbaix \& Eichhorn 1999).

HIP 2941: $M_{\mathrm{A}}=1.09 \pm 0.08 \quad M_{\odot}$ and $M_{\mathrm{B}}=0.87 \pm 0.07 M_{\odot}$ are relatively close to the solution of Duquennoy \& Mayor (1991). Adding some recent still unpublished radial velocities (Mayor \& Udry, priv. comm.), we obtain $\varpi=66.0 \pm 2.2$ mas, $M_{\mathrm{A}}=0.94 \pm 0.088 M_{\odot}$ and $M_{\mathrm{B}}=0.70 \pm 0.070 M_{\odot}$ which are more consistent with the spectral types (Gliese 1969).

HIP 10644: Although the standard deviations of the orbital parameters are quite small, we are puzzled by the shape of the cross-correlation dip obtained with CORAVEL (Fig. 1 in the paper by Duquennoy \& Mayor (1988a)). It suggests that the two profiles overlap almost every time. It is therefore difficult to imagine that precise radial velocities can be extracted for both components. The authors also assumed the orbit to be circular. Although the mass ratio is close to unity, their difference of magnitudes in blue is $2.0 \pm 0.2 \mathrm{mag}$. No information is given in the paper about the potential inconsistency of these two results $\left(\kappa \equiv \frac{M_{\mathrm{B}}}{M_{\mathrm{A}}+M_{\mathrm{B}}} \approx 0.5\right.$ and $\Delta m \approx 2 \mathrm{mag}$ ).

The simultaneous adjustment of the visual and spectroscopic data is a complete nightmare! In addition to the natural correlation between $\omega$ and $T$ due to the nearly circular orbit, there is another strong, -0.996 , correlation between $i$ and $\varpi$. Within the confidence interval on the inclination $\left(7^{\circ}\right.$-width), $\sin i$ ranges between 0.22 and 0.34 . Therefore, a small variation on $i$ implies a large variation on $\varpi$. That is the reason why our results are quite imprecise (and rather unreliable): $\varpi=136 \pm 30$ mas,
$M_{\mathrm{A}}=0.25 \pm 0.16 M_{\odot}$ and $M_{\mathrm{B}}=0.23 \pm 0.14 M_{\odot}$. The parallax after Hipparcos (ESA 1997) is $92.2 \pm 0.84$ mas is consistent neither with our value nor with the estimate of Van Altena et al. (1991).

HIP 12390: This system was previously known as an SB1 (Abt \& Levy 1976) with a somewhat uncertain period (Morbey \& Griffin 1987). Using CORAVEL, Duquennoy \& Mayor (1988a) could measure the spectra of both components and determined an SB2 orbit. As for HIP 10644, the two cross-correlation profiles (even at maximum separation) largely overlap. Using the visual orbit of Finsen (1970) (based on visual interferometric observations), Duquennoy \& Mayor derived $\varpi=66 \pm$ 10 mas (consistent with Gliese 1969), $M_{\mathrm{A}}=1.10 \pm$ $0.21 M_{\odot}$ and $M_{\mathrm{B}}=0.74 \pm 0.22 M_{\odot}$.

Hartkopf et al. (1989) determined a visual orbit based on almost ten years of speckle interferometric measurements. They did not look at the spectroscopic orbit and neither the parallax nor the masses were derived. One can, however, notice that the visual and spectroscopic orbits are not in perfect agreement. The solution of Hartkopf et al. does not agree with Finsen's either. Most of the visual interferometric observations used by Finsen (1970) have overestimated angular separations thus leading to an overestimated orbital parallax.

From the Hipparcos data and some photometric and astrometric assumptions, Martin \& Mignard (1998) derived $M_{\mathrm{A}}=1.886 \pm 0.171 M_{\odot}$ and $M_{\mathrm{B}}=0.990 \pm$ $0.092 M_{\odot}$. Their estimate for the secondary seems rather low for the announced spectral type F6V. Their mass ratio is also discrepant with respect to the spectroscopic one (Mazeh et al. 1992).

The spectroscopic data from Duquennoy \& Mayor (1988a) and all speckle interferometric observations (Hartkopf et al. 1997) yield $\varpi=34.9 \pm 3.9$ mas, $M_{\mathrm{A}}=2.39 \pm 0.74 M_{\odot}$ and $M_{\mathrm{B}}=1.55 \pm 0.48 M_{\odot}$. That parallax is consistent with the Hipparcos one: $36.99 \pm$ 1.76 mas.

HIP 20087: This system illustrates once again the improvement of the efficiency (Eichhorn 1989) when spectroscopic data are added to visual ones (Pourbaix \& Eichhorn 1999). Eichhorn \& Xu (1990) obtained 0.466 for the efficiency of the orbital parameters of the visual orbit. The present solution corresponds to 0.680 , thus confirming the weaker correlation between the parameters.

HIP 46404: Our parallax is quite discrepant with respect to the Hipparcos one: $32.01 \pm 1.02$. This discrepancy comes from the spectroscopy and, more precisely, from the two sets of radial velocities obtained by Duquennoy \& Mayor (1988a) when they disentangled the blended spectra of this system. However, by re-processing the Hipparcos Transit Data, Söderhjelm (1999) has lately revised the "Hipparcos" parallax down to $29.1 \pm 1.1$ mas 
Table 2. The semi-major axis of stars marked with ${ }^{*}$ is expressed in seconds of arc instead of mas. $\varpi$ stands for the parallax and $\kappa$ for the fractional mass $\left(\kappa=M_{\mathrm{B}} /\left(M_{\mathrm{A}}+M_{\mathrm{B}}\right)\right.$

\begin{tabular}{|c|c|c|c|c|c|c|c|c|c|c|c|c|}
\hline HIP & $\begin{array}{c}a \\
(\mathrm{mas})\end{array}$ & $\begin{array}{c}i \\
\left(^{\circ}\right)\end{array}$ & $\begin{array}{l}\omega \\
\left(^{\circ}\right)\end{array}$ & $\begin{array}{c}\Omega \\
\left(^{\circ}\right)\end{array}$ & $e$ & $\begin{array}{c}P \\
(\mathrm{yr})\end{array}$ & $\begin{array}{c}T \\
(\mathrm{Byr})\end{array}$ & $\begin{array}{c}V_{0} \\
\left(\mathrm{~km} \mathrm{~s}^{-1}\right)\end{array}$ & $\begin{array}{c}\varpi \\
(\mathrm{mas})\end{array}$ & $\kappa$ & $\begin{array}{c}M_{\mathrm{A}} \\
\left(M_{\odot}\right)\end{array}$ & $\begin{array}{c}M_{\mathrm{B}} \\
\left(M_{\odot}\right)\end{array}$ \\
\hline 677 & 24.0 & 105.6 & 257.4 & 284.4 & 0.535 & 0.26476 & 1988.5830 & -10.0 & 33.0 & 0.298 & 3.8 & 1.63 \\
\hline \pm & 0.13 & 0.23 & 0.31 & 0.21 & 0.0046 & 0.000012 & 0.00026 & 0.32 & 0.62 & 0.0065 & 0.22 & 0.074 \\
\hline 2941 & 667 & 77.6 & 317. & 291.8 & 0.235 & 25.09 & 1898.5 & 18.4 & 66 & 0.42 & 0.94 & 0.70 \\
\hline \pm & 7.0 & 0.44 & 2.8 & 0.47 & 0.0096 & 0.029 & 0.17 & 0.13 & 2.2 & 0.016 & 0.088 & 0.079 \\
\hline 4463 & 10.4 & 31. & 210 . & 70.0 & 0.008 & 0.31682 & 1992.86 & -10.4 & 13.1 & 0.474 & 2.6 & 2.3 \\
\hline \pm & 0.11 & 1.4 & 26 & 2.5 & 0.0054 & 0.000015 & 0.023 & 0.29 & 0.7 & 0.0091 & 0.35 & 0.31 \\
\hline 7580 & 324 & 96.6 & 251.6 & 159.6 & 0.798 & 28.8 & 1989.92 & 47.8 & 27. & 0.45 & 1.2 & 0.96 \\
\hline \pm & 5.4 & 0.33 & 0.67 & 0.73 & 0.0066 & 0.77 & 0.012 & 0.12 & 1.0 & 0.013 & 0.13 & 0.071 \\
\hline 8903 & 36.0 & 47.5 & 204.9 & 83.3 & 0.8801 & 0.292941 & 1981.55900 & -3.1 & 57.1 & 0.338 & 2.00 & 1.02 \\
\hline \pm & 0.16 & 0.54 & 0.33 & 0.27 & 0.00080 & $1.9 \mathrm{e}-06$ & $1.8 \mathrm{e}-05$ & 0.15 & 0.70 & 0.0026 & 0.053 & 0.032 \\
\hline 10064 & 8.03 & 130.0 & 118.1 & 245.2 & 0.433 & 0.085941 & 1984.91300 & 12.3 & 24.2 & 0.281 & 3.5 & 1.4 \\
\hline \pm & 0.061 & 0.52 & 0.66 & 0.67 & 0.0041 & $5.2 \mathrm{e}-07$ & $9.1 \mathrm{e}-05$ & 0.66 & 0.63 & 0.0093 & 0.25 & 0.1 \\
\hline 10644 & 9.8 & 163. & 171. & 37. & 0.011 & 0.027433 & 1992.812 & -6.6 & 136. & 0.470 & 0.2 & 0.2 \\
\hline \pm & 0.13 & 3.6 & 29 & 1.9 & 0.0055 & $4.7 \mathrm{e}-07$ & 0.0022 & 0.11 & 30 & 0.0098 & 0.16 & 0.14 \\
\hline $10952^{*}$ & 0.94 & 147. & 175. & 158. & 0.802 & 318. & 1989.2 & 10.0 & 17. & 0.4 & 1.1 & 0.7 \\
\hline \pm & 0.022 & 2.3 & 3.7 & 3.5 & 0.0072 & 17 & 0.14 & 0.98 & 1.4 & 0.12 & 0.32 & 0.26 \\
\hline 12390 & 106. & 24. & 41. & 270. & 0.230 & 2.651 & 1983.15 & 15.5 & 35. & 0.39 & 2.4 & 1.6 \\
\hline \pm & 1.5 & 2.5 & 3.4 & 2.8 & 0.0062 & 0.0018 & 0.011 & 0.19 & 3.9 & 0.015 & 0.74 & 0.48 \\
\hline 12623 & 53.1 & 127.0 & 89.9 & 49.3 & 0.663 & 0.90622 & 1993.3410 & -23.03 & 41.7 & 0.468 & 1.34 & 1.18 \\
\hline \pm & 0.66 & 0.76 & 0.30 & 0.57 & 0.0021 & $1.2 \mathrm{e}-05$ & 0.00028 & 0.042 & 0.89 & 0.0013 & 0.042 & 0.037 \\
\hline 14328 & 143.9 & 90.6 & 169.6 & 244.2 & 0.786 & 14.593 & 1947.279 & 3.2 & 14.7 & 0.423 & 2.5 & 1.86 \\
\hline \pm & 0.73 & 0.71 & 0.71 & 0.28 & 0.0038 & 0.0046 & 0.0083 & 0.13 & 0.19 & 0.0061 & 0.1 & 0.064 \\
\hline 20087 & 132.9 & 125.5 & 339. & 350.7 & 0.167 & 11.35 & 1977.74 & 37.86 & 17.5 & 0.45 & 1.9 & 1.5 \\
\hline \pm & 0.95 & 0.73 & 1.9 & 0.61 & 0.0044 & 0.021 & 0.056 & 0.099 & 0.61 & 0.017 & 0.14 & 0.2 \\
\hline 20661 & 100. & 125. & 272. & 215. & 0.716 & 6.28 & 1988.714 & 39.6 & 21. & 0.476 & 1.4 & 1.23 \\
\hline \pm & 3.1 & 1.6 & 1.1 & 1.7 & 0.0099 & 0.011 & 0.0074 & 0.11 & 1.1 & 0.0077 & 0.1 & 0.098 \\
\hline 24608 & 56.4 & 137.2 & 269. & 220.9 & 0.002 & 0.284809 & 1973.98 & 29.19 & 75.0 & 0.487 & 2.70 & 2.56 \\
\hline \pm & 0.11 & 0.19 & 26 & 0.31 & 0.0011 & $5.5 \mathrm{e}-06$ & 0.021 & 0.074 & 0.57 & 0.0029 & 0.066 & 0.043 \\
\hline 28360 & 3.38 & 75.0 & 319 & 294.5 & 0.000 & 0.010842 & 1975. & -15.8 & 40.7 & 0.499 & 2.4 & 2.44 \\
\hline \pm & 0.046 & 0.73 & - & 0.72 & 0.0075 & $7.2 \mathrm{e}-09$ & - & 0.51 & 0.77 & 0.0051 & 0.1 & 0.073 \\
\hline 38382 & 602. & 80.4 & 73.1 & 102.9 & 0.741 & 22.70 & 1985.92 & -21.3 & 62. & 0.48 & 0.93 & 0.9 \\
\hline \pm & 7.2 & 0.21 & 0.4 & 0.27 & 0.0070 & 0.027 & 0.021 & 0.16 & 2.6 & 0.018 & 0.082 & 0.12 \\
\hline 45170 & 115.4 & 124.1 & 350.7 & 317.6 & 0.433 & 2.7052 & 1982.690 & 49.82 & 49.4 & 0.49 & 0.89 & 0.85 \\
\hline \pm & 0.63 & 0.64 & 0.81 & 0.46 & 0.0034 & 0.00095 & 0.0040 & 0.076 & 0.62 & 0.004 & 0.029 & 0.026 \\
\hline 46404 & 406. & 84.1 & 172. & 150.8 & 0.25 & 34.5 & 1941.6 & 55.7 & 28. & 0.37 & 1.7 & 1.0 \\
\hline \pm & 5.7 & 0.7 & 6.2 & 0.46 & 0.012 & 0.32 & 0.50 & 0.19 & 3 & 0.042 & 0.64 & 0.25 \\
\hline 57565 & 7.33 & 49. & 277.16 & 320. & 0.000 & 0.196282 & 1979. & 0.5 & 13.3 & 0.476 & 2.2 & 2.0 \\
\hline \pm & 0.096 & 1.2 & - & 1.1 & 0.0052 & $1.6 \mathrm{e}-06$ & - & 0.21 & 0.45 & 0.0067 & 0.17 & 0.13 \\
\hline 65378 & 10.0 & 61. & 105.5 & 106. & 0.529 & 0.056233 & 1963.15100 & -6.3 & 40. & 0.493 & 2.5 & 2.5 \\
\hline \pm & 0.32 & 1.2 & 0.79 & 1.1 & 0.0052 & $3.7 \mathrm{e}-07$ & $7.6 \mathrm{e}-05$ & 0.38 & 1.8 & 0.0041 & 0.11 & 0.12 \\
\hline $71683 / 1^{*}$ & 17.59 & 79.23 & 231.8 & 204.82 & 0.519 & 79.90 & 1955.59 & -21.87 & 737. & 0.45 & 1.16 & 0.97 \\
\hline \pm & 0.028 & 0.046 & 0.15 & 0.087 & 0.0013 & 0.013 & 0.019 & 0.054 & 2.6 & 0.013 & 0.031 & 0.030 \\
\hline 73182 & 133. & 110. & 311. & 18. & 0.765 & 0.8429 & 1986.3660 & 28.1 & 155. & 0.420 & 0.51 & 0.37 \\
\hline \pm & 3.9 & 2.4 & 1.5 & 2.6 & 0.0083 & 0.00024 & 0.00082 & 0.25 & 6.5 & 0.0083 & 0.038 & 0.023 \\
\hline 75312 & 860. & 58.7 & 219.2 & 22.9 & 0.277 & 41.586 & 1892.317 & -7.41 & 54.9 & 0.472 & 1.19 & 1.05 \\
\hline \pm & 3.3 & 0.16 & 0.37 & 0.19 & 0.0011 & 0.008 & 0.031 & 0.054 & 0.97 & 0.0091 & 0.071 & 0.05 \\
\hline 85667 & 977. & 99.1 & 148. & 332.3 & 0.168 & 46.34 & 1870.0 & -77.18 & 61. & 0.481 & 0.98 & 0.90 \\
\hline \pm & 3.3 & 0.11 & 1.3 & 0.13 & 0.0025 & 0.021 & 0.16 & 0.069 & 1 & 0.0085 & 0.052 & 0.045 \\
\hline
\end{tabular}


Table 2. continued

\begin{tabular}{|c|c|c|c|c|c|c|c|c|c|c|c|c|}
\hline HIP & $\begin{array}{c}a \\
(\mathrm{mas})\end{array}$ & $\begin{array}{c}i \\
\left({ }^{\circ}\right)\end{array}$ & $\begin{array}{l}\omega \\
\left({ }^{\circ}\right)\end{array}$ & $\begin{array}{c}\Omega \\
\left({ }^{\circ}\right)\end{array}$ & $e$ & $\begin{array}{c}P \\
(\mathrm{yr})\end{array}$ & $\begin{array}{c}T \\
(\mathrm{Byr})\end{array}$ & $\begin{array}{c}V_{0} \\
\left(\mathrm{~km} \mathrm{~s}^{-1}\right)\end{array}$ & $\begin{array}{c}\varpi \\
(\mathrm{mas})\end{array}$ & $\kappa$ & $\begin{array}{c}M_{\mathrm{A}} \\
\left(M_{\odot}\right)\end{array}$ & $\begin{array}{c}M_{\mathrm{B}} \\
\left(M_{\odot}\right)\end{array}$ \\
\hline 87895 & 84. & 68. & 315. & 359. & 0.41 & 2.4143 & 1989.514 & -32.9 & 37. & 0.392 & 1.2 & 0.80 \\
\hline \pm & 3.0 & 2.4 & 1.9 & 1.4 & 0.011 & 0.00077 & 0.009 & 0.1 & 1.8 & 0.0079 & 0.11 & 0.055 \\
\hline $88601^{*}$ & 4.554 & 121.16 & 14.0 & 302.12 & 0.4992 & 88.38 & 1895.94 & -6.87 & 193. & 0.46 & 0.90 & 0.78 \\
\hline \pm & 0.0052 & 0.078 & 0.14 & 0.097 & 0.00039 & 0.017 & 0.017 & 0.077 & 4.2 & 0.013 & 0.074 & 0.040 \\
\hline 89937 & 123. & 74.8 & 299.9 & 50.5 & 0.414 & 0.7680 & 1984.835 & 31.90 & 122. & 0.416 & 1.03 & 0.73 \\
\hline \pm & 1.2 & 0.79 & 0.97 & 0.60 & 0.0083 & 0.00017 & 0.0015 & 0.14 & 2.1 & 0.0057 & 0.050 & 0.024 \\
\hline 91636 & 239. & 121. & 348. & 244. & 0.910 & 92.2 & 1980.82 & 17.8 & 8.4 & 0.54 & 1.22 & 1.47 \\
\hline \pm & 3.4 & 1.6 & 1.7 & 1.1 & 0.0020 & 0.41 & 0.015 & 0.50 & 0.22 & 0.018 & 0.086 & 0.085 \\
\hline 95995 & 86. & 144. & 356. & 243. & 0.360 & 1.3528 & 1985.271 & 11.31 & 59. & 0.482 & 0.8 & 0.8 \\
\hline \pm & 1.4 & 2.4 & 2.1 & 1.5 & 0.0078 & 0.00072 & 0.0047 & 0.099 & 4.1 & 0.0050 & 0.15 & 0.14 \\
\hline 96683 & 26.9 & 80.8 & 34. & 251.0 & 0.542 & 1.18872 & 1936.169 & 5.2 & 14.2 & 0.491 & 2.46 & 2.39 \\
\hline \pm & 0.75 & 0.63 & 1.3 & 0.86 & 0.0063 & $4.1 \mathrm{e}-05$ & 0.0017 & 0.14 & 0.42 & 0.0032 & 0.055 & 0.045 \\
\hline 98416 & 150. & 6. & 142. & 147. & 0.592 & 4.895 & 1982.81 & 30.02 & 11. & 0.45 & 53. & 43. \\
\hline \pm & 2.7 & 20.0 & 4.3 & 3.0 & 0.0094 & 0.0054 & 0.031 & 0.094 & 38. & 0.022 & 530. & 440. \\
\hline 99376 & 458. & 115.3 & 159. & 321.7 & 0.492 & 85.2 & 1970.4 & -43.2 & 18. & 0.44 & 1.2 & 0.9 \\
\hline \pm & 4.4 & 0.42 & 1 & 0.52 & 0.0033 & 0.12 & 0.18 & 0.20 & 1.0 & 0.044 & 0.23 & 0.17 \\
\hline 99473 & 3.20 & 142. & 211. & 95. & 0.59 & 0.046884 & 1964.1060 & -28.0 & 14. & 0.444 & 3.2 & 2.5 \\
\hline \pm & 0.075 & 3.1 & 3.3 & 3.2 & 0.013 & $2.9 \mathrm{e}-07$ & 0.00012 & 0.52 & 1.2 & 0.0053 & 0.67 & 0.54 \\
\hline 103655 & 690. & 44. & 129. & 122. & 0.72 & 29.5 & 1976.88 & -33.97 & 45 & 0.24 & 3. & 1.0 \\
\hline \pm & 50 & 8.9 & 3.3 & 6.5 & 0.014 & 0.66 & 0.048 & 0.095 & 14 & 0.057 & 2.7 & 0.65 \\
\hline 104858 & 232. & 99.0 & 8. & 203.8 & 0.440 & 5.703 & 1981.47 & -15.85 & 55.0 & 0.484 & 1.19 & 1.12 \\
\hline \pm & 1.8 & 0.43 & 1.0 & 0.29 & 0.0046 & 0.0070 & 0.012 & 0.074 & 0.67 & 0.0043 & 0.034 & 0.032 \\
\hline 104987 & 12.0 & 153. & 120. & 34. & 0.004 & 0.27056 & 1990.96 & -17.6 & 18. & 0.47 & 2.3 & 2.0 \\
\hline \pm & 0.14 & 3.1 & 30 & 3.8 & 0.0072 & $4.5 \mathrm{e}-05$ & 0.022 & 0.21 & 2.0 & 0.012 & 0.77 & 0.66 \\
\hline 108917 & 72. & 68. & 273. & 85. & 0.50 & 2.241 & 1970.992 & -10.7 & 38. & 0.26 & 1.0 & 0.36 \\
\hline \pm & 1.7 & 1.4 & 1.1 & 1.9 & 0.021 & 0.0027 & 0.0092 & 0.34 & 2.1 & 0.017 & 0.13 & 0.051 \\
\hline 111170 & 71.6 & 67. & 352. & 262.4 & 0.38 & 1.7253 & 1979.330 & -9.72 & 38. & 0.353 & 1.4 & 0.78 \\
\hline \pm & 0.76 & 1.3 & 1.7 & 0.55 & 0.011 & 0.00097 & 0.0069 & 0.097 & 1.2 & 0.0098 & 0.14 & 0.051 \\
\hline 111528 & 141. & 60. & 324. & 294. & 0.36 & 22.3 & 1985.2 & 6.86 & 13.3 & 0.487 & 1.22 & 1.16 \\
\hline \pm & 4.5 & 2.1 & 2.2 & 1.8 & 0.013 & 0.11 & 0.10 & 0.074 & 0.65 & 0.0072 & 0.094 & 0.091 \\
\hline 114576 & 204. & 104. & 130. & 106. & 0.41 & 29.1 & 1983.2 & 34.5 & 16.7 & 0.39 & 1.3 & 0.84 \\
\hline \pm & 5.6 & 2.4 & 2.1 & 2.9 & 0.015 & 0.21 & 0.12 & 0.12 & 0.71 & 0.010 & 0.11 & 0.066 \\
\hline
\end{tabular}

which yields a mass sum of $2.54 \pm 0.41 M_{\odot}$ thus confirming our results.

HIP 73182: This system has all the features that made it a "tough case" for the Hipparcos reduction teams. The semi-major axis has the same order of magnitude as the parallax. Moreover, the orbital period is close to one year. The consequence is a large confidence interval on the parallax: $133.63 \pm 33.56$ mas. At this level of confidence, the consistency of this parallax with ours is just fortuitous! Söderhjelm (1999) has lately re-processed the Hipparcos Transit Data and derived $\varpi=169.7 \pm 1.0$ mas and a mass sum of $0.83 \pm 0.11 M_{\odot}$. His "Hipparcos" parallax and ours do no longer agree although the mass sums are very consistent.

HIP 75312: Our initial parallax was quite discrepant with respect to the Hipparcos one $53.70 \pm 1.24$ mas. From the Hipparcos data, Söderhjelm (1999) derived $\varpi=$ $53.5 \pm 0.9$ mas and a mass sum of $2.41 \pm 0.14 M_{\odot}$. Because we strongly believed that the way the CORAVEL data of this LWSB had been disentangled by the Geneva group was responsible for a large part of this discrepancy, we asked S. Udry to slightly tune the input parameters of the disentangling procedure, which he did. With these revised radial velocities (the procedure was applied to all RVs from Geneva), we now obtain: $\varpi=54.9 \pm 0.97$ mas, $M_{\mathrm{A}}=1.19 \pm 0.071 M_{\odot}$ and $M_{\mathrm{B}}=1.05 \pm 0.050 M_{\odot}$.

HIP 89937: Our adjustment yields $M_{\mathrm{A}}=1.03 \pm$ $0.050 M_{\odot}$ and $M_{\mathrm{B}}=0.73 \pm 0.024 M_{\odot}$. The masses after Tomkin et al. (1987) as well as ours do not correspond well with what one expects for a F7V and a late G-type dwarf and the masses by Breakiron \& Gatewood (1974) remain more likely.

HIP 103655: From the visual observations kept at US Naval Observatory and the radial velocities of A and B by Duquennoy \& Mayor (1988b), we cannot derive any stable solution. Using the mass ratio of Fekel et al. (1978), we can compute the systemic velocities of $\mathrm{Aa}-\mathrm{Ab}$ from the radial velocities of the two components. When these new "measurements" are added to the set of radial velocities, the shape of the objective function (Pourbaix 1998) starts 


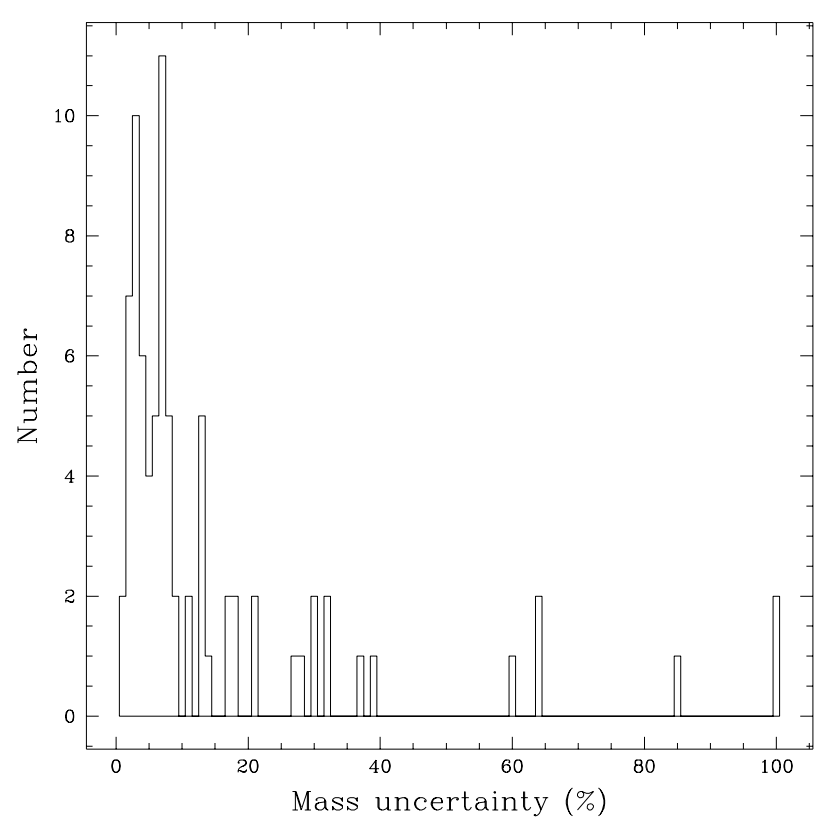

Fig. 1. Distribution of the uncertainty (in per cent) of the individual masses after this study

exhibiting some larger variations and, hence, a global minimum neighborhood. Nevertheless, this region remains valueless. We obtain $\varpi=45 \pm 14$ mas, $M_{\mathrm{A}}=3.1 \pm$ $2.7 M_{\odot}$ and $M_{\mathrm{B}}=1.0 \pm 0.65 M_{\odot}$. The only result we share with Russell \& Gatewood (1980) seems to be the period: $29.5 \pm 0.6$ years.

Our parallax is totally discrepant with respect to the Hipparcos one, 66.21 \pm 2.54 mas, the latter being consistent with the result by Russell \& Gatewood (1980). Since both the spectroscopic and visual date are of poor quality, it was expected that the Hipparcos observations would improve the visual part. Söderhjelm (1999) has lately obtained a parallax of $66 \pm 1.8$ mas and a mass sum of $1.38 \pm$ $0.34 M_{\odot}$ from the Hipparcos Transit Data.

\section{What about the precision of the masses?}

We want to figure out whether or not the masses we obtain are precise and also how much better (or worse) our values are with respect to former results. The histogram in Fig. 1 answers the first question.

Among the 80 stars, 64 have a mass whose uncertainty is lower than 20 per cent (e.g., HIP $7580 \mathrm{~A}$ and B). This is the large majority, but let us recall that 20 per cent of uncertainty is still large for subsequent investigations (e.g., calibration of a mass-luminosity relation). We know the mass of 52 stars (65 per cent of the sample) at a level of uncertainty lower than ten per cent (e.g., HIP 14328 $\mathrm{A}$ and B). And, finally, at an uncertainty level lower than or equal to five per cent, we still have 25 stars (e.g.,
HIP 8903 A and B). With modern interferometers (CHARA-ARRAY, NPOI, TPI, ...), this number should promptly grow.

To generate the histogram in Fig. 1, we used a maximum threshold of one hundred per cent (threshold reached by HIP $98416 \mathrm{~A}$ and B). The uncertainty is actually much larger than the plotted value. Indeed, nothing reliable could be derived for that system due to the very large correlation between $i$ and $\varpi$.

We should also stress on the fact that the above uncertainties are consistent with the actual uncertainties of the observations. Indeed, we check the consistency of the guessed standard deviations on the observations with those of the $(\mathrm{O}-\mathrm{C})$ after the minimization (Pourbaix 1998). In case of discrepancies, the input values were revised and the minimization was repeated. We can thus guarantee that the uncertainties we derive for the masses are realistic. Even if we limit the sample to the main-sequence stars, we seldom reach the 2\%-accuracy required (Andersen 1991; Andersen 1997) for constraining theoretical models. However, the mass of some non main-sequence stars is precise (hopefully accurate) enough (e.g. $\gamma$ Per) to point out some underlying problems (Pourbaix 1999).

The evolution of the uncertainty of the masses is more difficult to analyze. Indeed, an uncertainty of ten per cent on a wrong result is worse than fifteen per cent on a good one (e.g., HIP 677 B). We thus restrict the comparison on stars for which the mass we obtain lies in a $2 \sigma_{\mathrm{M}}$-interval centered on the published value of the mass (we use the published $\sigma_{\mathrm{M}}$ ). This restriction leads to a drastic reduction of the number of stars: we have 42 stars left (instead of 80 ).

The plot in Fig. 2 gives the evolution of the uncertainty on the 42 masses. The former uncertainties are those computed from the pairs $\left(M, \sigma_{\mathrm{M}}\right)$ given in the papers listed in Table 1 . We reduced the uncertainty for 23 stars. The worst evolution concerns HIP 104987 for which our uncertainties are 33 and 32 per cent whereas Armstrong et al. (1992) announced 14 and 11 per cent. However, in some cases, it may be judicious to re-assess the quoted errors because large variations may be caused by previous overestimation of the obtained precision.

The best improvement concerns both components of HIP 108917 for which we decreased the uncertainty from 60 down to thirteen and fourteen per cent. However, since there is a correlation between $a, i$ and $\varpi$, the actual improvement might be not that important. We also recall that the orbital parallax for that system is not consistent with the Hipparcos result.

\section{Conclusions}

Although we have mainly used already published data, we did revise the parallaxes and the individual 


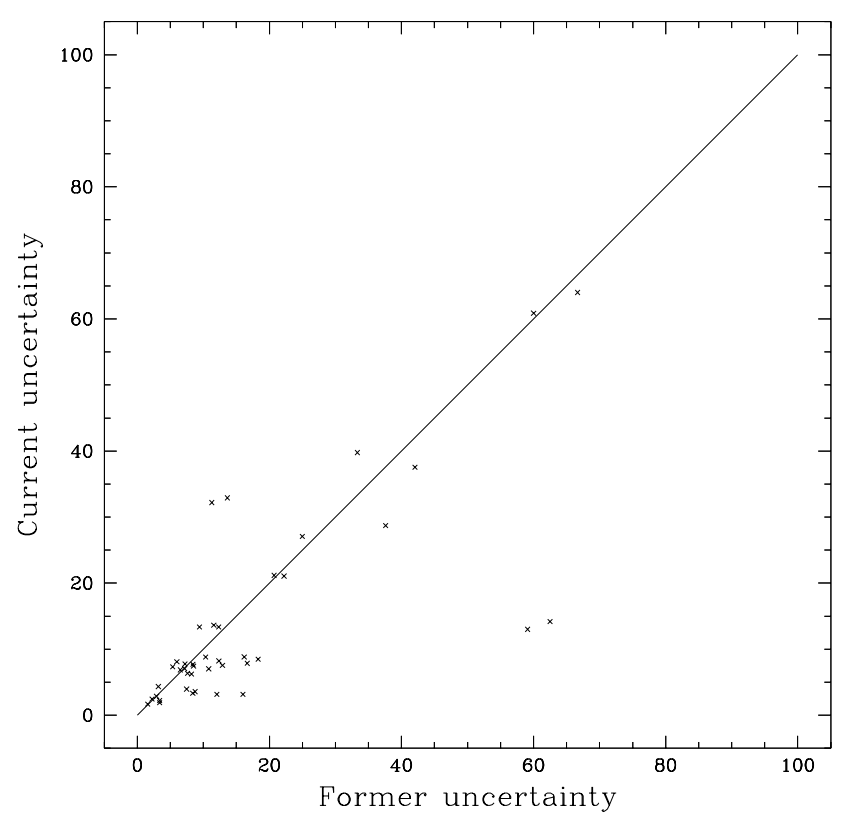

Fig. 2. Evolution of the uncertainty (in per cent) of the individual masses after this study

masses of some of those systems. That was sometimes possible thanks to the integration of unpublished observations kindly provided by the authors (e.g., HIP 14328). However, just fitting published data more efficiently provided considerable improvement (e.g., HIP 677) as shown in Fig. 2. For some systems, we simply confirmed an available solution (e.g., HIP 104858). But, even in those cases, we usually supplied the first simultaneous orbital solution and, therefore, the first homogeneous standard deviations based on all observations. Usually, that yielded narrower confidence intervals, more consistent with the estimated errors on the observations.

Being hypothesis-free, orbital parallaxes are also useful to check the reliability of other determinations (e.g., spectroscopic parallaxes). For instance, the agreement between the Hipparcos results and those we derive is quite good.

Acknowledgements. I thank Patricia Lampens for her careful reading of a very preliminary version of this manuscript and for her valuable suggestions. I also thank my former colleagues from the Royal Observatory of Belgium and from the University of Liege for the fruitful discussions we had about stellar masses. Michel Mayor, Stephane Udry and Roger Griffin are thanked for supplying some radial velocities prior to their publication. This research has made use of the Washington Double Star Catalog maintained at the U.S. Naval Observatory and of the Simbad database operated at CDS, Strasbourg, France.

\section{References}

Abt H.A., Levy S.G., 1976, ApJS 30, 273

Aikman G.C.L., 1976, Publ. Dom. Ap. Obs. 14, 379

Andersen J., 1991, A\&AR 3, 91

Andersen J., 1997, in Bedding T.R., Booth A.J., Davis J. (eds.), Fundamental Stellar Properties: The Interaction between Observation and Theory. Kluwer Academic Publishers

Armstrong J.T., Hummel C.A., Quirrenbach A., et al., 1992, AJ 104, 2217

Armstrong J.T., Mozurkewich D., Rickard L.J., et al., 1998, ApJ 496, 550

Bagnuolo W.G. Jr., Sowell J.R., 1989, AJ 96, 1056

Bahng J.D.R., 1958, ApJ 128, 572

Barlow D.J., Fekel F.C., Scarfe C.D., 1993, PASP 105, 476

Barlow D.J., Scarfe C.D., Fekel F.C., 1998, AJ 115, 2555

Batten A.H., Fisher W.A., Fletcher J.M., Hill G., 1983, PASP 95, 768

Batten A.H., Fletcher J.M., 1991, PASP 103, 546

Batten A.H., Fletcher J.M., Campbell B., 1984, PASP 96, 903

Batten A.H., Fletcher J.M., Fisher W.A., et al., 1982, PASP 94, 860

Batten A.H., Fletcher J.M., Hill G., et al., 1991, PASP 103, 294

Berman L., 1932, Lick Obs. Bull. 16, 24

Boden A.F., Koresko C.D., Van Belle G.T., et al., 1999, ApJ 515,356

Breakiron L.A., Gatewood G., 1974, PASP 86, 448

Carney B.W., Latham D.W., Laird J.B., Aguilar L.A., 1994, AJ 107, 2240

Cesco C.U., Struve O., 1946, ApJ 104, 282

Christy J.W., Walker R.L. Jr., 1969, PASP 81, 643

Colacevich A., 1941, Oss. e. Mem. Acetri 59, 16

Colavita M.M., Wallace J.K., Hines B.E., et al., 1999, ApJ 510, 505

Cowley A., Cowley C., Jaschek M., Jaschek C., 1969, AJ 74, 375

Delfosse X., Forveille T., Beuzit J.-L., et al., 1999, A\&A 344, 897

Dommanget J., Nys O., 1982, Comm. Obs. Roy. Belgique Ser. B (124)

Duquennoy A., 1987, A\&A 178, 114

Duquennoy A., Mayor M., 1988a, A\&A 195, 129

Duquennoy A., Mayor M., 1988b, A\&A 200, 135

Duquennoy A., Mayor M., 1991, A\&A 248, 485

Duquennoy A., Mayor M., Griffin R.F., et al., 1988, A\&AS 75, 167

Duquennoy A., Mayor M., Halbwachs J.L., 1991, A\&AS 88, 281

Ebbighausen E.G., 1953, Publ. Dom. Ap. Obs. XI, 277

Eichhorn H., 1989, Bull. Astron. Inst. Czechosl. 40 (6), 394

Eichhorn H.K., Xu Y.-L., 1990, ApJ 358, 575

ESA, 1997, The Hipparcos and Tycho Catalogues, ESA SP-1200

Fehrenbach C., Prevot L., 1961, J. Obs. 44, 83

Fekel F. Jr., Bopp B.W., Lacy C.H., 1978, AJ 83, 1445

Fekel F.C., 1992, in McAlister H.A., Hartkopf W.I. (eds.), IAU Coll. 135, ASP Conf. Ser. 32, 89

Fernie J.D., 1969, JRASC 63, 113

Finsen W.S., 1970, IAU Comm. 26 Circ. Inf. 52 
Gliese W., 1969, Veroeff. Astron. Recher Inst. Heidelberg 22, 1 Gordon K., 1946, ApJ 104, 282

Gorza W.L., Heard J.F., 1971, Pub. David Dunlap Obs. 3, 99

Griffin R.F., 1984, Observatory 104, 143

Griffin R.F., Griffin R.E.M., Snyder L.F., et al., 1994, IAPPP Comm. 57, 31

Hartkopf W.I., Mason B.D., McAlister H.A., 1996, AJ 111, 370

Hartkopf W.I., McAlister H.A., Franz O.G., 1989, AJ 98, 1014

Hartkopf W.I., McAlister H.A., Mason B.D., 1997, Third Catalog of Interferometric Measurements of Binary Stars, Center for High Angular Resolution Astronomy, Georgia State University, Atlanta, Georgia, 30303-3083

Heintz W.D., 1988, JRASC 82, 140

Hilditch R.W., Edwin R.P., Milligan H.M., McFadzean A.D., 1988, Observatory 108, 228

Hoffleit D., Jaschek C., 1982, Bright Star Catalogue, Yale University Observatory, New Haven, 4th edition

Hummel C.A., Armstrong J.T., Buscher D.F., et al., 1995, AJ 110,376

Hummel C.A., Armstrong J.T., Quirrenbach A., et al., 1994, AJ 107, 1859

Hummel C.A., Armstrong J.T., et al., 1993, AJ 106, 2486

Hummel C.A., Mozurkewich D., Armstrong J.T., et al., 1998, AJ 116, 2536

Koresko C.D., Van Belle G.T., Boden A.F., et al., 1998, ApJ 509, L45

Malbet F., Berger J.P., Colavita M.M., et al., 1998, ApJ 507, L149

Mariotti J.M., Perrier C., Duquennoy A., Duhoux P., 1990, A\&A 230, 77

Martin C., Mignard F., 1998, A\&A 330, 585

Mason B.D., McAlister H.A., Hartkopf W.I., 1996, AJ 112, 276

Mazeh T., Goldberg D., Duquennoy A., Mayor M., 1992, ApJ 401, 265

McAlister H., 1997, in Docobo J.A., Elipe A., McAlister H. (eds.), Visual double stars: formation, dynamics and evolutionary tracks. Kluwer Academic Publishers, p. 3
McAlister H.A., Hartkopf W.I., Mason B.D., et al., 1995, AJ 110,366

McClure R.D., 1983, PASP 95, 201

Morbey C.L., Griffin R.F., 1987, ApJ 317, 343

Pan X., Shao M., Colavita M.M., et al., 1992, ApJ 384, 624

Pan X.P., Shao M., Colavita M.M., et al., 1990, ApJ 356, 641

Pike C.D., 1978, MNRAS 184, 265

Popper D.M., 1980, ARA\&A 18, 115

Pourbaix D., 1998, A\&AS 131, 377

Pourbaix D., 1999, A\&A 348, 127

Pourbaix D., Eichhorn H., 1999, A\&AS 136, 419

Pourbaix D., Neuforge-Verheecke C., Noels A., 1999, A\&A 344, 172

Prieur J.-L., Oblak E., Lampens P., et al., 2000 (in preparation)

Rach R.A., Herbig G.H., 1961, ApJ 133, 143

Roman N.G., 1952, ApJ 116, 122

Rosvick J.M., Scarfe C.D., 1991, MNRAS 252, 68

Rufener F., 1976, A\&AS 26, 275

Russell J., Gatewood G., 1980, AJ 85, 1270

Smith B., 1948, ApJ 108, 504

Söderhjelm S., 1999, A\&A 341, 121

Stephenson C.B., Sanwal N.B., 1969, AJ 74, 689

Struve O., Pogo A., 1928, ApJ 67, 336

Tokovinin A.A., 1993, SvAL 19, 73

Tomkin J., McAlister H.A., Hartkopf W.I., 1987, AJ 93, 1236

Tomkin J., Pan X., McCarthy J.K., 1995, AJ 109, 780

Tomkin J., Tran H., 1987, AJ 94, 1664

Torres G., 1995, PASP 107, 524

Torres G., Stefanik R.P., Latham D.W., 1997a, ApJ 474, 256

Torres G., Stefanik R.P., Latham D.W., 1997b, ApJ 479, 268

Van Altena W.F., Lee J.T.-L., Hoffleit E.D., 1991, General Catalogue of Trigonometric Stellar Parallaxes: A preliminary version, Yale University Observatory, New Haven

Vickers C.R., Scarfe C.D., 1976, PASP 88, 944

Worley C.E., Douglass G.G., 1996, Washington Double Star Catalog of observations, U.S. Naval Observatory 